\title{
Incompressible SPH Model for Simulating Violent Free-Surface Fluid Flows
}

\author{
Ryszard Staroszczyk \\ Institute of Hydro-Engineering, Polish Academy of Sciences, ul. Kościerska 7, 80-328 Gdańsk, Poland, \\ e-mail: rstar@ibwpan.gda.pl
}

(Received September 11, 2014; revised October 21, 2014)

\begin{abstract}
In this paper the problem of transient gravitational wave propagation in a viscous incompressible fluid is considered, with a focus on flows with fast-moving free surfaces. The governing equations of the problem are solved by the smoothed particle hydrodynamics method (SPH). In order to impose the incompressibility constraint on the fluid motion, the so-called projection method is applied in which the discrete SPH equations are integrated in time by using a fractional-step technique. Numerical performance of the proposed model has been assessed by comparing its results with experimental data and with results obtained by a standard (weakly compressible) version of the SPH approach. For this purpose, a plane dam-break flow problem is simulated, in order to investigate the formation and propagation of a wave generated by a sudden collapse of a water column initially contained in a rectangular tank, as well as the impact of such a wave on a rigid vertical wall. The results of simulations show the evolution of the free surface of water, the variation of velocity and pressure fields in the fluid, and the time history of pressures exerted by an impacting wave on a wall.
\end{abstract}

Key words: gravity water wave, free-surface, incompressible flow, Lagrangian description, smoothed particle hydrodynamics

\section{Notation}

$\begin{array}{ll}a & - \text { reference particle label, } \\ b & - \text { neighbouring particle label, } \\ h & - \text { kernel smoothing length, } \\ H & - \text { initial water column height, } \\ L & - \text { initial water column length, } \\ m & - \text { mass, } \\ N & - \text { number of particles in a support domain, } \\ p & - \text { pressure, } \\ R & - \text { kernel support domain radius, } \\ t & - \text { time, } \\ v & - \text { velocity vector, }\end{array}$




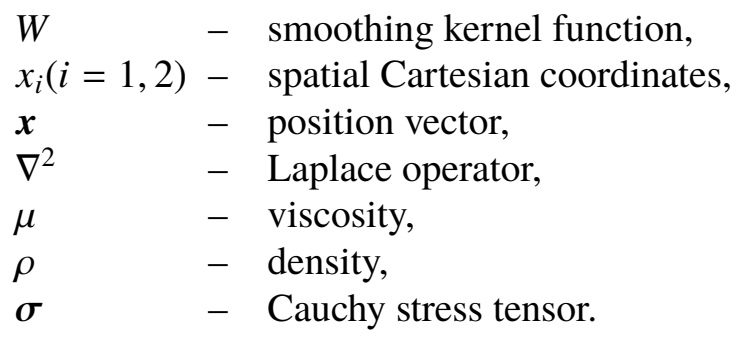

\section{Introduction}

Conventionally, fluid dynamics problems that involve moving free surfaces have been solved numerically by grid-based methods. The classical approach consists in solving physical governing equations in the Eulerian (spatial) frame. Typical representatives of such an approach include the finite difference method and the finite volume method. However, owing to its inherent features, the Eulerian description has serious limitations, and often fails when attempting to simulate violent flows, such as wave breaking, green water overtopping, sloshing, or water impact on solid structures; that is, when the position of the free surface changes rapidly, or fragmentation of the fluid body occurs. A more successful approach to such complex phenomena is based on the Lagrangian (material) description, in which a discrete grid is attached to selected particles of the material. Such a grid follows the deformation of the fluid, and, since the fluid boundaries are material surfaces that do not move in the Lagrangian coordinate frame, the solution of boundary-value problems is easier than in the case of the Eulerian formulation. Typically, the Lagrangian methods are applied within the framework of the finite element method (Ramaswamy and Kawahara 1987, Radovitzky and Ortiz 1998, Staroszczyk 2007, 2009). A more recent approach consists in combining the advantages of the spatial and material description, forming the basis of so-called Arbitrary Lagrangian-Eulerian (ALE) method. In this approach, a purely Eulerian method is applied to one part of the fluid domain, whereas the other (near moving boundaries) is treated by a purely Lagrangian method, which makes this approach computationally very complicated and expensive (Braess and Wriggers 2000, Souli and Zolesio 2001, Rabier and Medale 2003).

Yet, despite much progress achieved in recent years in the field of computational fluid dynamics, it is recognized that the above mesh-based methods cannot successfully deal with certain classes of phenomena, in which discontinuities develop and subsequently evolve within the fluid, leading to the separation of some of its parts from the main body. In such cases, it is more natural, and usually more straightforward in numerical implementations, to apply one of the so-called particle methods. Rigourous foundations of such meshless methods were formulated in the 1990s in the field of solid mechanics (Belytschko et al 1996, 1998), though some ideas of such methods appeared as early as in the mid 1950s, when particle-in-cell (PIC) and marker-and-cell (MAC) methods were developed (Harlow 2004, Harlow and Welch 1965). 
One of the most popular meshless methods is the smoothed particle hydrodynamics (SPH) approach. The method was invented by Lucy (1977) and Gingold and Monaghan (1977) and was originally used only in the field of astrophysics. However, in the mid 1990s the SPH method, owing to its attractive features, such as the natural capability of dealing with large displacements and deformations, attracted the attention of the solid mechanics community (Johnson et al 1996, Randles and Libersky 1996). Ever since, the interest in the method has been growing, and it has found application in many branches of applied mechanics and engineering, including hydrodynamics as well as hydro- and off-shore engineering (Monaghan 1996, Shao and Lo 2003, Colagrossi and Landrini 2003, Gómez-Gesteira et al 2005, Dalrymple and Rogers 2006, Antoci et al 2007, Shao 2010, Staroszczyk 2011).

Basically, two versions of the SPH method have been developed and used in practice. In the original, and still most common approach, referred to as weakly compressible SPH (WCSPH), the fluid is treated as a compressible medium, with the compressibility modulus adjusted artificially in such a way that typical fluctuations of the fluid density are of order $1 \%$. An advantage of this approach is that the resulting discrete equations can be solved by using computationally fast explicit time-stepping schemes. Examples of the application of the WCSPH method can be found in Monaghan (1992), Colagrossi and Landrini (2003), Antoci et al (2007), Staroszczyk (2010), Cummins et al (2012) and many other publications. An alternative approach is based on the assumption (very common in the theory of gravity waves) that the fluid is incompressible. A characteristic feature of this approach, termed incompressible SPH (ISPH), is the necessity of solving a Poisson equation for pressure at each time step in order to enforce incompressibility of the medium. This significantly increases the cost of computations, and requires special techniques for solving the discrete equations in the time domain. Usually a fractional-step projection method is applied, in which, at each time step, the velocity field is first calculated without enforcing incompressibility, and then the intermediate velocity field is projected onto a divergent-free space to satisfy the incompressibility constraint (Chorin 1968, Cummins and Rudman 1999). Some examples of the application of the ISPH approach are presented by Lo and Shao (2002), Shao (2006) and Hu and Adams (2007).

It is known from computational practice that fluid pressure fields predicted by the WCSPH method exhibit some noisiness, and discrete particles tend to cluster near free surfaces of the fluid. These negative features are due to the stiff character of the equation of state used in the method. To some extent the results can be improved by performing a so-called correction of the SPH interpolating functions based on the moving least squares (MLS) technique (Colagrossi and Landrini 2003, Dalrymple and Rogers 2006, Staroszczyk 2010). It turns out, however, that an even greater improvement in terms of the smoothness of the fluid pressure fields obtained from simulations can be achieved by employing the ISPH method, as demonstrated by Rafiee et al (2012). In many engineering problems the accurate determination of the water pressure field, that is the forces exerted by water on structures, is of paramount 
significance. For this reason, the ISPH method has been selected in the present work to simulate violent free-surface flows in which the interaction of water with rigid walls has a very dynamic nature. In order to assess how the more complex ISPH method performs against the simpler standard WCSPH approach, the two models are used to simulate a classical two-dimensional dam-break flow problem; the latter has been frequently used for testing numerical implementations of various discrete methods (Colagrossi and Landrini 2003, Quecedo et al 2005, Szydłowski and Zima 2006, Chang et al 2011). The predictions of the ISPH and WCSPH models are compared with each other and, in addition, some comparisons are also made with experimental data available from the literature. Hence, results showing the time evolution of the water free surface and the velocity and pressure fields in water are presented, and, in particular, the time history of pressures exerted by a surging wave during its impact on a rigid vertical wall is illustrated.

The paper is structured as follows. First, equations defining the problem to be solved are presented in Section 2. The following Section 3 deals with the discrete formulation of the equations involved, together with the description of the fractional time-stepping scheme used to integrate the ISPH equations. Then, in Section 4, results of numerical simulations are presented, and, finally, some conclusions are given in Section 5.

\section{Problem Formulation}

A plane water flow problem with a free surface is considered, in which the fluid is treated as isotropic, linearly viscous and incompressible. It is assumed that the flow occurs under isothermal conditions and is entirely due to the action of gravity. The problem is solved in rectangular Cartesian coordinates, with origin $O$ and the coordinate axes $x_{i}(i=1,2)$, see Fig. 1 . Let $t$ denote time, then the current position of a fluid particle is described by the position vector $\boldsymbol{x}$ with components $x_{i}(t)$.

The law of mass conservation expressed in the adopted coordinate frame is expressed in a local form by the continuity equation

$$
\frac{\mathrm{D} \rho}{\mathrm{D} t}+\rho \nabla \cdot \boldsymbol{v}=0,
$$

where $\mathrm{D} / \mathrm{D} t$ denotes the material (convected) time derivative, $\rho$ is the fluid density, $\boldsymbol{v}$ is the fluid velocity vector, and $\nabla$ stands for the nabla operator. Note that the form of the continuity equation (1) corresponds to that for a compressible fluid, even though the incompressible flow is actually analysed. The purpose of this is that the variations of the fluid density at each discrete particle during a predictor step are used to enforce incompressibility during a correction step of the time integration scheme, which will be described further in Section 3.2.

The linear momentum conservation balance, in the presence of body forces, for a linearly viscous (Newtonian) and incompressible liquid, is given by the Navier-Stokes equation of the form: 


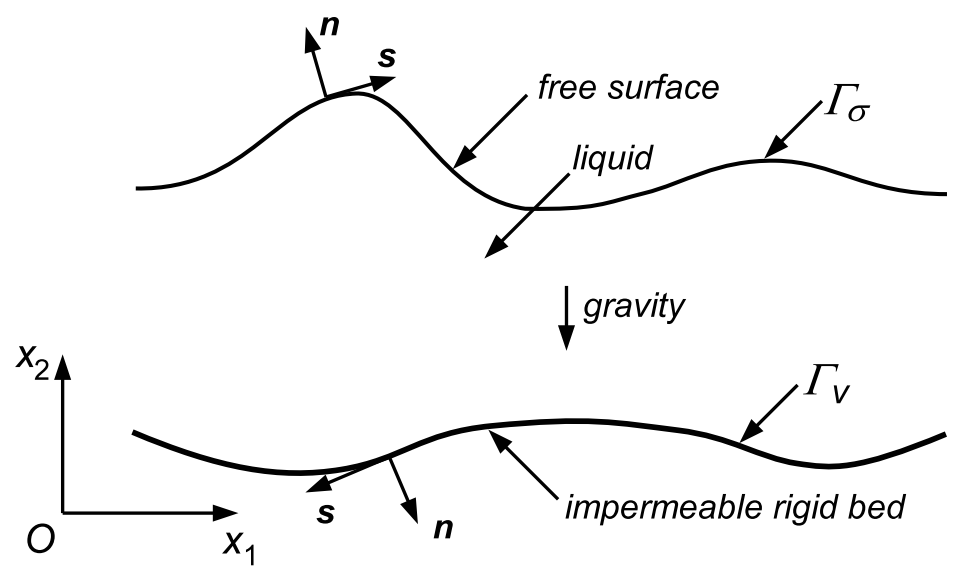

Fig. 1. Plane free-surface liquid flow problem definition

$$
\rho \frac{\mathrm{D} \boldsymbol{v}}{\mathrm{D} t}=-\nabla p+\mu \nabla^{2} \boldsymbol{v}+\rho \boldsymbol{g},
$$

where $p$ is pressure, $\mu$ is the fluid viscosity, $\nabla^{2}$ is the vector Laplace operator, and $\boldsymbol{g}$ denotes the body force vector. In the problem investigated, the only body force is that due to gravity. The viscosity term is included in the motion equation (2) solely in order to improve the stability of the numerical time-integration scheme, as it is realized that viscosity effects are negligibly small in the physical problem considered here.

To complete the problem statement, initial and boundary conditions must be specified. It is supposed that the fluid motion starts at time $t=0$, and at $t \leq 0$ the fluid is at rest, that is, the fluid velocities are zero, and the initial stress in the fluid is that of the hydrostatic pressure. Hence, the initial conditions are defined by

$$
t=0: \quad \boldsymbol{v}=\mathbf{0} \quad \text { and } \quad \boldsymbol{\sigma}=-p \boldsymbol{I},
$$

where $\sigma$ denotes the Cauchy stress tensor and $\boldsymbol{I}$ is the unit tensor.

The boundary conditions are adopted in the standard form. Hence, the fluid free surface is assumed to be traction free, following from the tacit assumption that all stresses in the fluid are measured relative to the atmospheric pressure, treated as constant and equal to zero. Thus, on the free surface, denoted as $\Gamma_{\sigma}$, we have

$$
\boldsymbol{n} \cdot(\boldsymbol{\sigma} \boldsymbol{n})=0 \quad \text { and } \quad \boldsymbol{s} \cdot(\boldsymbol{\sigma} \boldsymbol{n})=0, \quad \text { on } \quad \Gamma_{\sigma},
$$

where $\boldsymbol{n}$ and $\boldsymbol{s}$ are the unit vectors normal and tangential, respectively, to the boundary. At solid boundaries, $\Gamma_{v}$, treated as rigid and impermeable walls, free-slip conditions are adopted. This simplification implies that boundary layer effects are not accounted for. Accordingly, kinematic conditions at the solid boundaries are expressed as 


$$
\boldsymbol{v} \cdot \boldsymbol{n}=0 \quad \text { on } \quad \Gamma_{v},
$$

meaning that the velocity component tangential to $\Gamma_{v}$ is unconstrained.

\section{Incompressible SPH Methodology}

In the SPH method, a continuum is represented by a collection of discrete particles, each of which carries, in a Lagrangian sense, information on the local physical properties of the system under consideration. Since no topological connectivity for neighbouring particles is needed, the method has a fully mesh-free character, which makes it very suitable for dealing with large deformations, material discontinuities, etc. In order to approximate field variables between arbitrarily distributed discrete particles, and to calculate spatial derivatives at each location, special interpolating functions are used. These functions, known as smoothing kernels in the SPH, define weightings with which individual particles contribute to the approximated value of a dependent variable at a given spatial or material point. A characteristic feature of the SPH smoothing kernel is that it has non-zero values only in a small domain, called the kernel support. Most often, the kernel has a shape of a circle or sphere centred at a given material particle or spatial point. Thus, the number of particles involved in approximation is limited (typically 20 to 50 in two-dimensional problems), which facilitates fast computations. Through the use of the smoothing kernels, the field functions are expressed by integrals, which in turn are approximated by summations over all particles contained within the kernel support. The spatial derivatives of the field functions are evaluated similarly, by summation formulae, which involve spatial derivatives of the smoothing kernel functions (the latter functions, and their derivatives, are usually defined by analytical expressions).

Let $a$ and $b$ be labels of a pair of neighbouring material particles. Then, the value of any field variable $f(\boldsymbol{x})$ at particle $a$ (called a reference particle), situated at position $\boldsymbol{x}_{a}$, is evaluated by means of a kernel function, $W$, centred at this particle, by the following interpolation formula

$$
f_{a}=\sum_{b=1}^{N} V_{b} f_{b} W_{a b} .
$$

In the above relation, $f_{a}=f\left(\boldsymbol{x}_{a}\right)$ and $f_{b}=f\left(\boldsymbol{x}_{b}\right)$ are the discrete values of $f$ at particles $a$ and $b$, respectively, $V_{b}$ is the volume of particle $b$, and $N$ is the number of particles currently located within the kernel support domain. The abbreviation $W_{a b}$, typical of the SPH convention, denotes the value of the kernel $W$, centred at $\boldsymbol{x}_{a}$, at the point $\boldsymbol{x}_{b}$. That is,

$$
W_{a b}=W\left(r_{a b}, h\right), \quad r_{a b}=\left|\boldsymbol{x}_{a b}\right|=\left|\boldsymbol{x}_{a}-\boldsymbol{x}_{b}\right|,
$$

where $r_{a b}$ is the distance between particles $a$ and $b$. The parameter $h$ in (7) denotes the kernel smoothing length, which defines the resolution of discretization, that is, the 
average number of discrete particles involved in kernel interpolations. Spatial differentiation of the function $W$ gives the gradient of the kernel, denoted by $\nabla_{a} W_{a b}$, and defined by

$$
\nabla_{a} W_{a b}=\frac{\boldsymbol{x}_{a b}}{r_{a b}} \frac{\partial W\left(r_{a b}, h\right)}{\partial r_{a b}} .
$$

The latter relation will be used in the next section to define SPH approximations to differential operators.

\subsection{SPH Equations}

There is a variety of possible discrete approximations to differential operators that can be used in the SPH formulations (Monaghan 1992, Li and Liu 2004). Usually, the best accuracy of conservation laws is achieved by applying forms that possess certain symmetry properties with respect to particle indices $a$ and $b$. In this work, forms recommended by Monaghan (1992) are employed. Hence, the first-order operators, the gradient of a scalar function $f$ and the divergence of a vector function $f$ are respectively approximated at particle $a$ by the formulae:

$$
\left(\frac{\nabla f}{\rho}\right)_{a}=\sum_{b=1}^{N} m_{b}\left(\frac{f_{a}}{\rho_{a}^{2}}+\frac{f_{b}}{\rho_{b}^{2}}\right) \nabla_{a} W_{a b},
$$

and

$$
\left(\frac{\nabla \cdot \boldsymbol{f}}{\rho}\right)_{a}=-\sum_{b=1}^{N} m_{b} \boldsymbol{f}_{a b} \cdot \nabla_{a} W_{a b},
$$

where $m_{b}$ denotes the mass of particle $b, \rho_{b}$ is its density, and $\boldsymbol{f}_{a b}=\boldsymbol{f}_{a}-\boldsymbol{f}_{b}$.

The approximation of the Laplace operator can be derived in a straightforward manner by the successive application of the gradient and divergence operators defined above. It has been found, however, that the resulting formula, because of the presence of the second-order derivative of the kernel function, is very sensitive to the distribution of particles (Cummins and Rudman 1999, Shao and Lo 2003). More efficient is a hybrid approach in which the Laplacian approximation is constructed by combining the first-order derivative with a finite-difference approximation. The resulting formula for the SPH Laplace operator has then the form (Shao and Lo 2003):

$$
\nabla \cdot\left(\frac{\nabla f}{\rho}\right)_{a}=\sum_{b=1}^{N} m_{b} \frac{8}{\left(\rho_{a}+\rho_{b}\right)^{2}} \frac{f_{a b} \boldsymbol{x}_{a b} \cdot \nabla_{a} W_{a b}}{r_{a b}^{2}+\eta^{2}},
$$

with the notation $\boldsymbol{x}_{a b}=\boldsymbol{x}_{a}-\boldsymbol{x}_{b} . \eta$ is a small parameter, usually adopted as $\eta=0.1 h$, introduced to regularize the denominator of the last term in (11). 
The application of the approximations (9)-(11) to the continuity and momentum equations, (1) and (2) respectively, yields the following SPH expressions for a discrete material particle $a$ :

$$
\frac{\mathrm{d} \rho_{a}}{\mathrm{~d} t}=\sum_{b=1}^{N} m_{b} \boldsymbol{v}_{a b} \cdot \nabla_{a} W_{a b}
$$

and

$$
\frac{\mathrm{d} v_{a}}{\mathrm{~d} t}=-\sum_{b=1}^{N} m_{b}\left(\frac{p_{a}}{\rho_{a}^{2}}+\frac{p_{b}}{\rho_{b}^{2}}+\Pi_{a b}\right) \nabla_{a} W_{a b}+\boldsymbol{g}_{a} .
$$

In the latter equation, the term $\Pi_{a b}$ defines the viscous force between particles $a$ and $b$, and, in view of the approximation formula (11), is given by

$$
\Pi_{a b}=-\frac{8 \mu}{\left(\rho_{a}+\rho_{b}\right)^{2}} \frac{\boldsymbol{v}_{a b} \boldsymbol{x}_{a b}}{r_{a b}^{2}+\eta^{2}} .
$$

Shao and Lo (2003) consider a more general relation for $\Pi_{a b}$, admitting different viscosities at particles $a$ and $b$; in the present analysis, however, it is supposed that the viscosity $\mu$ has a constant value throughout the liquid body.

The ordinary differential equations (12) and (13), describing interaction between a given particle and its neighbours, must be supplemented by an equation describing the particle trajectory $\boldsymbol{x}(t)$, which is simply expressed by

$$
\frac{\mathrm{d} \boldsymbol{x}_{a}}{\mathrm{~d} t}=\boldsymbol{v}_{a} .
$$

\subsection{Time Integration of the SPH Equations}

The discrete representations of the continuity, momentum and displacement equations, (12), (13) and (15), form a set of differential equations that must be integrated in the time domain, given the initial conditions stated generally in equation (3). In order to satisfy the fluid incompressibility constraint, $\nabla \cdot \boldsymbol{v}=0$, the equations are typically integrated by applying a two-step method proposed by Chorin (1968), subsequently called the fractional step method, or the projection method. Several variants of this method have been developed since its origination. In the present study, we follow an approach which is similar to that of Shao and Lo (2003) and Rafiee et al (2012).

Accordingly, at a given time step of length $\Delta t$, advancing the solution from time instant $t_{n}$ to $t_{n+1}$, the equations are first integrated explicitly without enforcing the fluid incompressibility. That is, in expression (13), approximating the the Navier-Stokes equation (2), the pressure gradient term is ignored, so that only the viscous stress and body force terms are retained. Hence, an intermediate velocity of particle $a$ is calculated as

$$
\boldsymbol{v}_{a}^{*}=\boldsymbol{v}_{a}^{n}-\Delta t\left(\sum_{b=1}^{N} m_{b} \Pi_{a b}+\boldsymbol{g}_{a}\right),
$$


the particle is shifted to its intermediate position $\boldsymbol{x}_{i}^{*}$, see (15), by

$$
\boldsymbol{x}_{a}^{*}=\boldsymbol{x}_{a}^{n}+\Delta t \boldsymbol{v}_{a}^{*}
$$

and, using the shifted particle positions, the fluid density is calculated from the relation (Rafiee et al 2012)

$$
\rho_{a}^{*}=\sum_{b=1}^{N} m_{b} \widetilde{W}_{a b},
$$

where $\widetilde{W}_{a b}$ denotes the kernel functions smoothed out by the Shepard filtering formula (Dalrymple and Rogers 2006)

$$
\widetilde{W}_{a b}=\frac{W_{a b}}{\sum_{b=1}^{N} V_{b} W_{a b}}, \quad V_{b}=\frac{m_{b}}{\rho_{b}} .
$$

Note that the values of the kernel functions $W_{a b}$ at all neighbouring particles $b$ of a reference particle $a$ will change slightly due to the changes in distances $\left|\boldsymbol{x}_{a}^{*}-\boldsymbol{x}_{b}^{*}\right|$ between all the particles involved.

The intermediate fluid densities $\rho_{a}^{*}$ determined from (18) deviate from the initial constant value $\rho_{0}$. Therefore, a second corrective step is performed in order to adjust the density to its value $\rho_{0}$ at the beginning, and the end, of the time step. For this purpose, the pressure term in the momentum equation (2), in the SPH form represented by (13), is now used (with the other terms being omitted) in order to update the intermediate velocity $\boldsymbol{v}_{a}^{*}$. Hence, by expressing (2) for particle $a$ in the approximate form

$$
\frac{\boldsymbol{v}_{a}^{n+1}-\boldsymbol{v}_{a}^{*}}{\Delta t}=-\frac{1}{\rho^{*}} \nabla p_{a}^{n+1},
$$

and by applying the divergence operator, we have

$$
\frac{1}{\Delta t}\left(\nabla \cdot \boldsymbol{v}_{a}^{n+1}-\nabla \cdot \boldsymbol{v}_{a}^{*}\right)=-\nabla\left(\frac{1}{\rho_{a}^{*}} \nabla \cdot p_{a}^{n+1}\right) .
$$

In the above equations, $\boldsymbol{v}_{a}^{n+1}$ and $p_{a}^{n+1}$ denote, respectively, the velocity and pressure at the end of the time step, at the instant $t_{n+1}$. Since it is required that at the end of the step the incompressibility condition $\nabla \cdot \boldsymbol{v}_{a}^{n+1}=0$ is fulfilled, equation (21) becomes

$$
\frac{\nabla \cdot \boldsymbol{v}_{a}^{*}}{\Delta t}=\nabla \cdot\left(\frac{1}{\rho_{a}^{*}} \nabla p_{a}^{n+1}\right)
$$

which is the Poisson equation for pressure with a source term involving the divergence of the intermediate velocity vector. The latter quantity, $\nabla \cdot \boldsymbol{v}_{a}^{*}$, is determined 
from the continuity equation (1), in the SPH form given by (12). Accordingly, after approximating the time derivative in equation (1) by the finite difference, one obtains

$$
\nabla \cdot \boldsymbol{v}_{a}^{*}=\frac{\rho_{a}^{*}-\rho_{0}}{\rho_{a}^{*} \Delta t} .
$$

Combining now equations (22) and (23) gives

$$
\nabla \cdot\left(\frac{1}{\rho_{a}^{*}} \nabla p_{a}^{n+1}\right)=\frac{\rho_{a}^{*}-\rho_{0}}{\rho_{a}^{*}(\Delta t)^{2}},
$$

where the Laplacian on the left-hand side is expressed in the SPH form by (11). Thus, by applying this approximation, equation (24) becomes

$$
\sum_{b=1}^{N} m_{b} \frac{8}{\left(\rho_{a}^{*}+\rho_{b}^{*}\right)^{2}} \frac{p_{a b}^{n+1} \boldsymbol{x}_{a b}^{*} \cdot \nabla_{a} W_{a b}}{\left(r_{a b}^{*}\right)^{2}+\eta^{2}}=\frac{\rho_{a}^{*}-\rho_{0}}{\rho_{a}^{*}(\Delta t)^{2}} .
$$

The above is an implicit relation for the pressures $p^{n+1}$ at all particles of the discrete system (recall that $p_{a b}$ stands for $p_{a}-p_{b}$ ). Thus, it represents a system of linear algebraic equations, with a sparse matrix of coefficients.

With pressures at all particles calculated from (25), the final velocity at particle $a$ at the end of the current time step can be determined from equation (20), with the gradient operator approximation given by (9), to yield

$$
\boldsymbol{v}_{a}^{n+1}=\boldsymbol{v}_{a}^{*}-\Delta t \sum_{b=1}^{N} m_{b}\left(\frac{p_{a}^{n+1}}{\rho_{a}^{2}}+\frac{p_{b}^{n+1}}{\rho_{b}^{2}}\right) \nabla_{a} W_{a b},
$$

and the position of particle $a$ at time $t_{n+1}$ is

$$
\boldsymbol{x}_{a}^{n+1}=\boldsymbol{x}_{a}^{n}+\Delta t\left(\frac{\boldsymbol{v}_{a}^{n}+\boldsymbol{v}_{a}^{n+1}}{2}\right) .
$$

\section{Numerical Simulations}

The numerical SPH model presented in the preceding section has been employed to simulate a plane dam-break problem, in which violent water flow is induced by the collapse of a water column, resulting from an instantaneous removal of a gate lock containing the liquid in a reservoir. Such a classical problem, however idealized, is often used as a benchmark test for assessing the performance and robustness of numerical techniques for solving computational fluid dynamics problems. Two cases, sketched in Figure 2, are considered. In the first case, water is released into a channel with a dry bed (i.e. the channel contains no water prior to the dam failure), whereas in the second case there is a shallow layer of water in the channel. In order to assess the accuracy of the SPH model predictions, some of the numerical results obtained have 
(a)
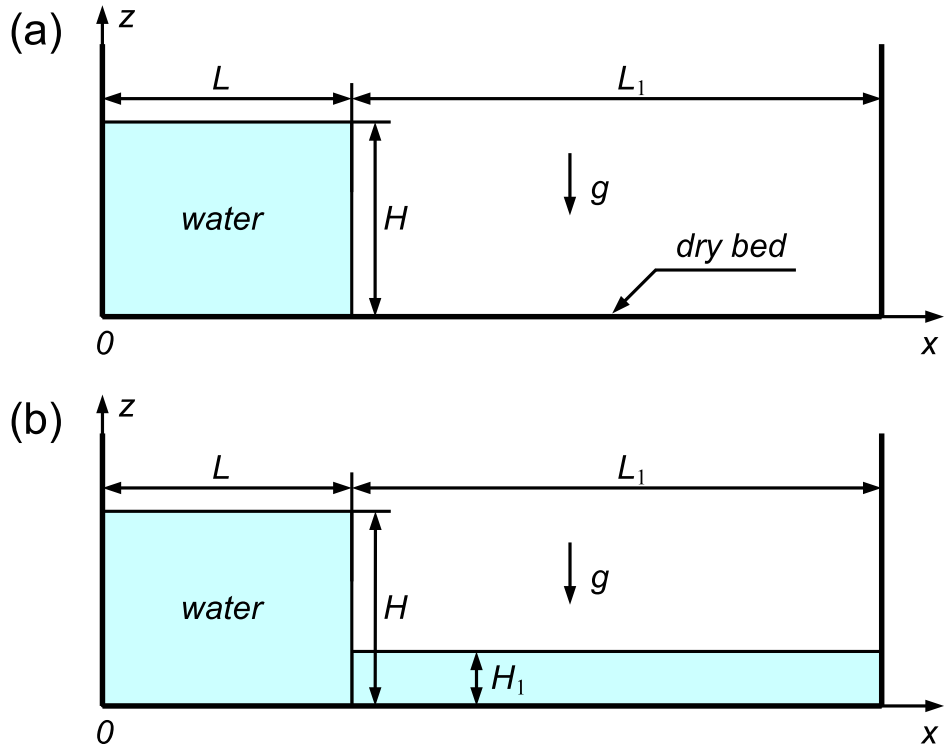

Fig. 2. Dam-break problem definitions: geometric parameters and coordinate axes

been compared with experimental measurements by Martin and Moyce (1952) for the dry-bed case shown in Fig. 2(a). Therefore, the specific geometric dimensions have been adopted to agree with those occurring in the corresponding laboratory tests.

The simulations for the case (a) in Fig. 2 were conducted for the geometric aspect ratio $L / H=1$, which is one of the dimensionless ratios investigated by Martin and Moyce (1952). Hence, the parameters $H=L=1.0 \mathrm{~m}$, defining the initial geometry of the water reservoir, were used in calculations. The water domain was discretized by adopting a uniform grid of particles in the initial fluid configuration, with equal spacings in the $x$ and $z$ directions. The inter-particle spacing was taken as $d=(1 / 60)$ $\mathrm{m}$; so that the SPH model consisted of $60 \times 60=3600$ particles. In the simulations, the kernel function proposed by Morris (1996) was used, which is a polynomial approximation of the Gaussian kernel function (Monaghan 1992). The kernel support domain radius was taken as $R=4 d$, which implied that, in the initial configuration, a typical particle inside the fluid domain had about 50 interacting neighbours. The evolution equations were integrated with the time step of length $\Delta t=2 \times 10^{-4} \mathrm{~s}$. The density of water was adopted as $\rho_{0}=10^{3} \mathrm{~kg} / \mathrm{m}^{3}$, and its viscosity as $\mu=1.01 \times 10^{-3}$ $\mathrm{Ns} \mathrm{m}^{-2}$. The initial conditions assumed at $t=0$ were those of the hydrostatic equilibrium, that is, $v_{x}=v_{z}=0$ and $p=\rho_{0} g(H-z)$. At all solid boundaries, the vertical walls at $x=0$ and $x=L+L_{1}$ and the horizontal bed $z=0$, free-slip conditions were adopted. These conditions were implemented in the numerical code by introducing so-called ghost (fictitious) particles outside the walls (Cummins and Rudman 1999), in such a way that all their physical properties (mass, density, volume, velocity and 
pressure) mirror the corresponding properties of real particles distributed (at a given time instant) along the walls inside the water domain.
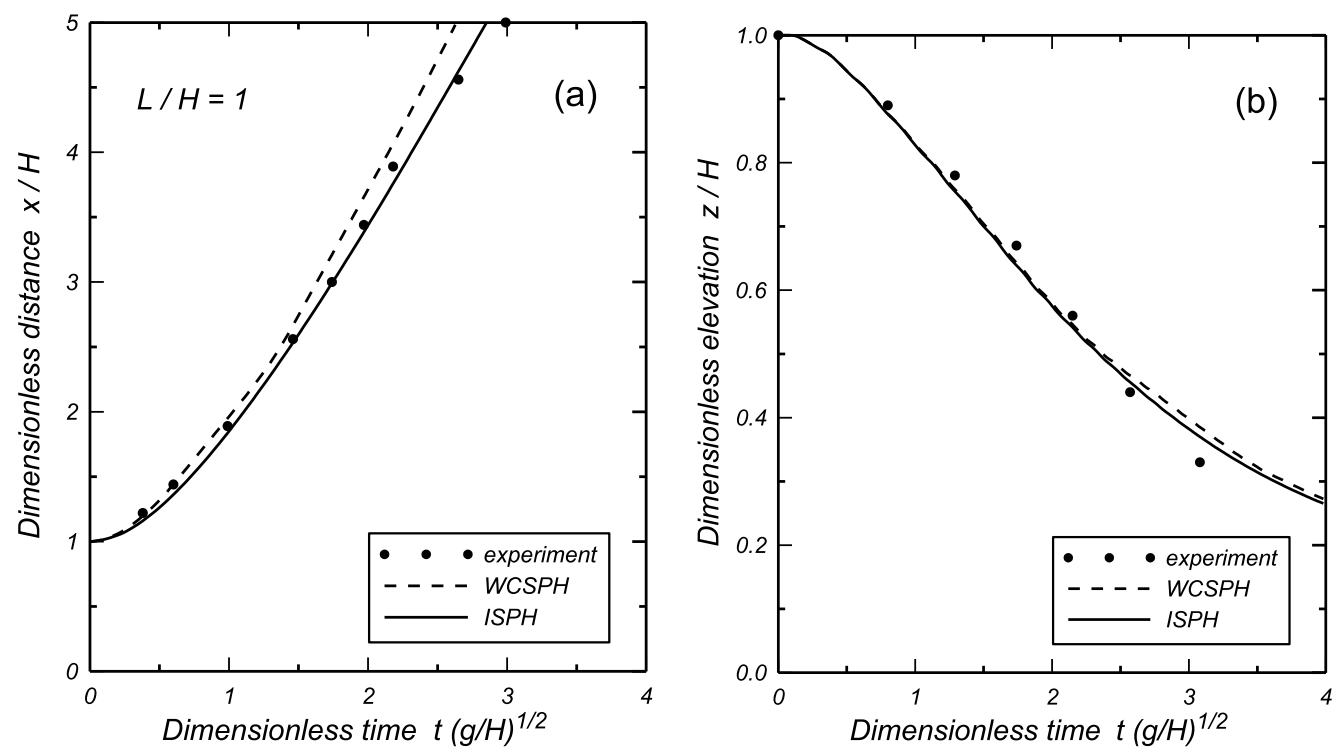

Fig. 3. Time evolution of the surging wave front position $x / H$ (a), and the free-surface elevation $z / H$ at the wall $z=0(\mathrm{~b})$, for $L / H=1$. Comparison of numerical (lines) and experimental (solid dots) results

Figure 3 illustrates the evolution of the surging wave front position, $x / H$, and the free-surface elevation, $z / H$, at the wall $x=0$, for the aspect ratio $L / H=1$, as functions of dimensionless time $t \sqrt{g / H}$. The numerical results are compared with empirical data obtained by Martin and Moyce (1952), represented by the solid dots. Shown are the predictions of the standard weakly compressible (WCSPH) method, represented by the dashed lines, and of the present incompressible (ISPH) method, represented by the solid lines. It is seen that the application of the incompressible version of the $\mathrm{SPH}$ improves the results, though this improvement is more pronounced for the prediction of the wave front position, whereas the free-surface elevations given by the two methods are very similar. Similarly good agreement between the ISPH results and the experimental data was achieved for the aspect ratio $L / H=0.5$.

The plots presented in Figures 4, 5 and 6 show the evolution of the water domain for a water tank of the dimensions $L=H=1 \mathrm{~m}$ with time elapsing from the dam-break instant $t=0$. First, in the series of plots in Figure 4, flow over a dry bed is illustrated. It is seen that discrete particles are distributed in a regular and consistent pattern, and the particles on the top form a smooth free surface. This indicates that the SPH model has been properly constructed and yields reliable results, at least in terms of the qualitative behaviour of the liquid. Only after a relatively long time of simulation the numerical solution starts to deteriorate near the leading edge of the 

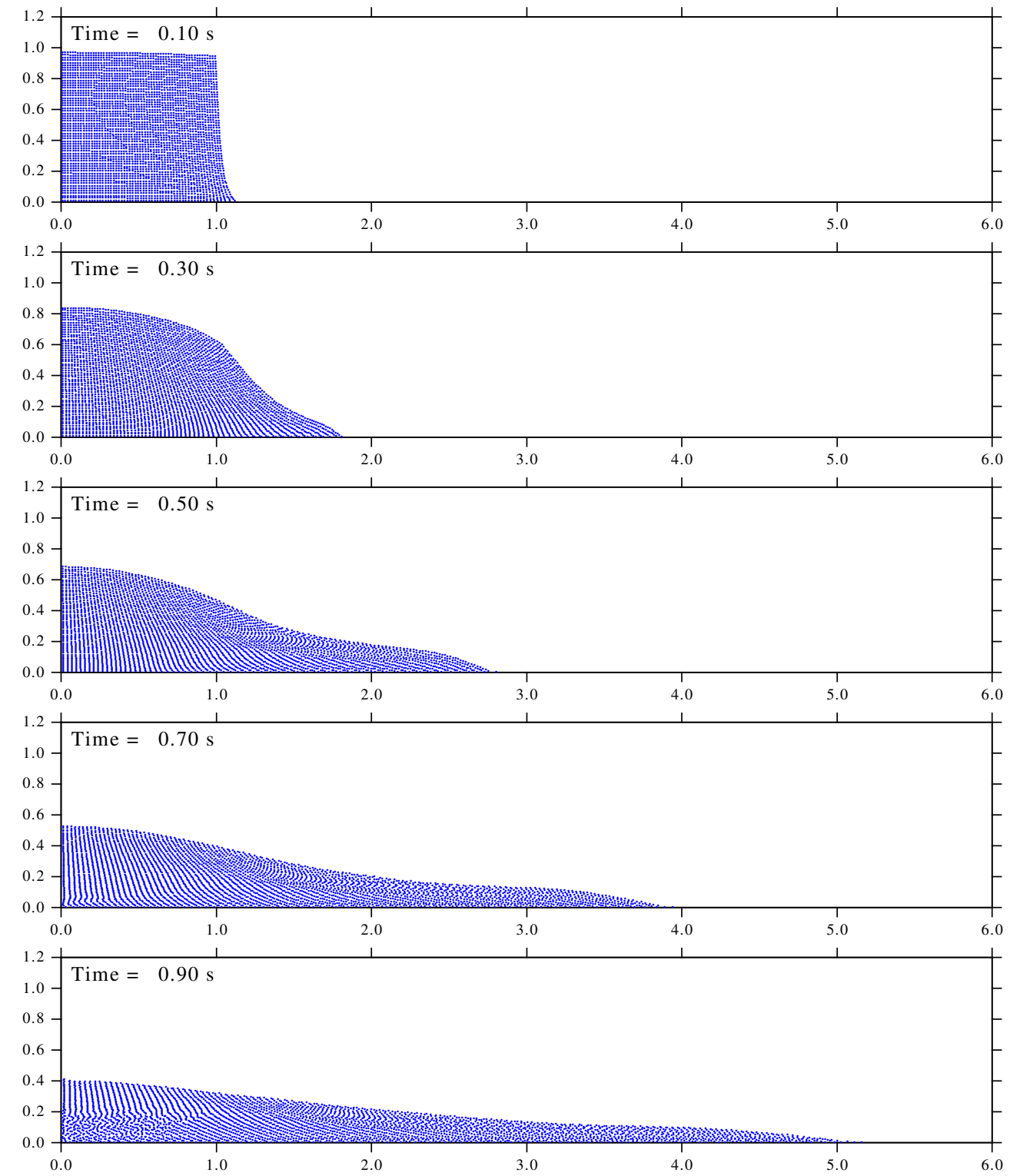

Fig. 4. Time evolution of the water domain during dam-break flow over a dry bed ( $L=H=1 \mathrm{~m}, 3600$ discrete particles used in the simulations)

wave, which is due to the fact that there are too few discrete particles across the very shallow water domain to accurately interpolate field variables.

In the next two sets of plots, in Figures 5 and 6, dam-break flow over a layer of fluid is illustrated. Figure 5 presents the results of simulations for a shallower layer, for which $H_{1} / H=0.2$, whereas the following Figure 6 illustrates the case of a deeper layer, defined by $H_{1} / H=0.4$. One can see in both figures that the presence of an 


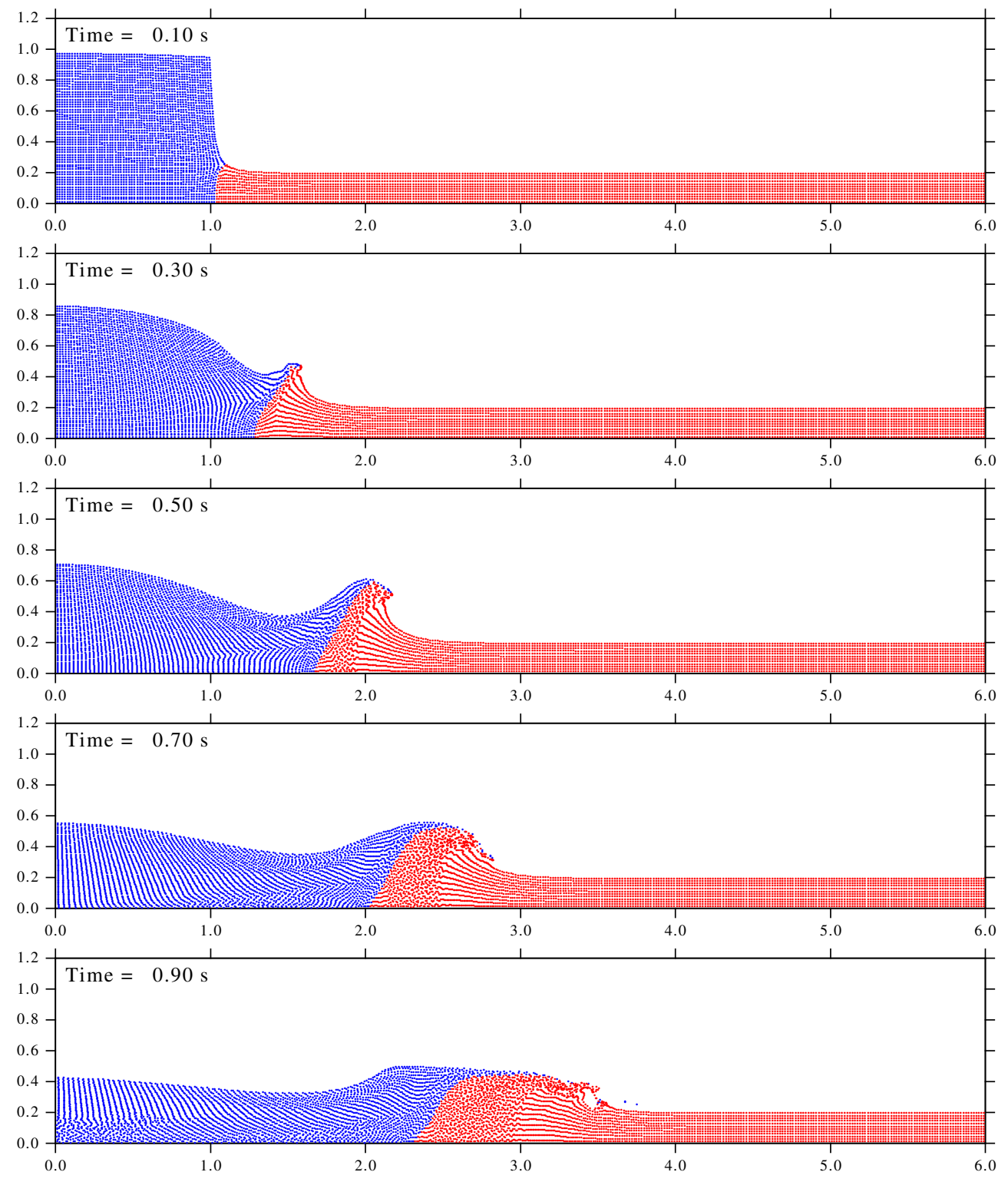

Fig. 5. Time evolution of the water domain during dam-break flow over a dry bed ( $L=H=1 \mathrm{~m}, H_{1}=0.2 \mathrm{~m}, L_{1}=5 \mathrm{~m}, 7200$ discrete particles used in the simulations)

ambient layer of water, due to its dynamic interaction with the surging wave, gives rise to a different behaviour of the fluid system. Two features can be noticed in the plots. First, that the layer of water outside the dam can effectively constrain the water from the dam reservoir by preventing it from moving far away from the dam wall. Another feature is the possibility of forming a breaking wave in the region near the moving interface of the two fluids, which can be seen in the case of the shallow water, 


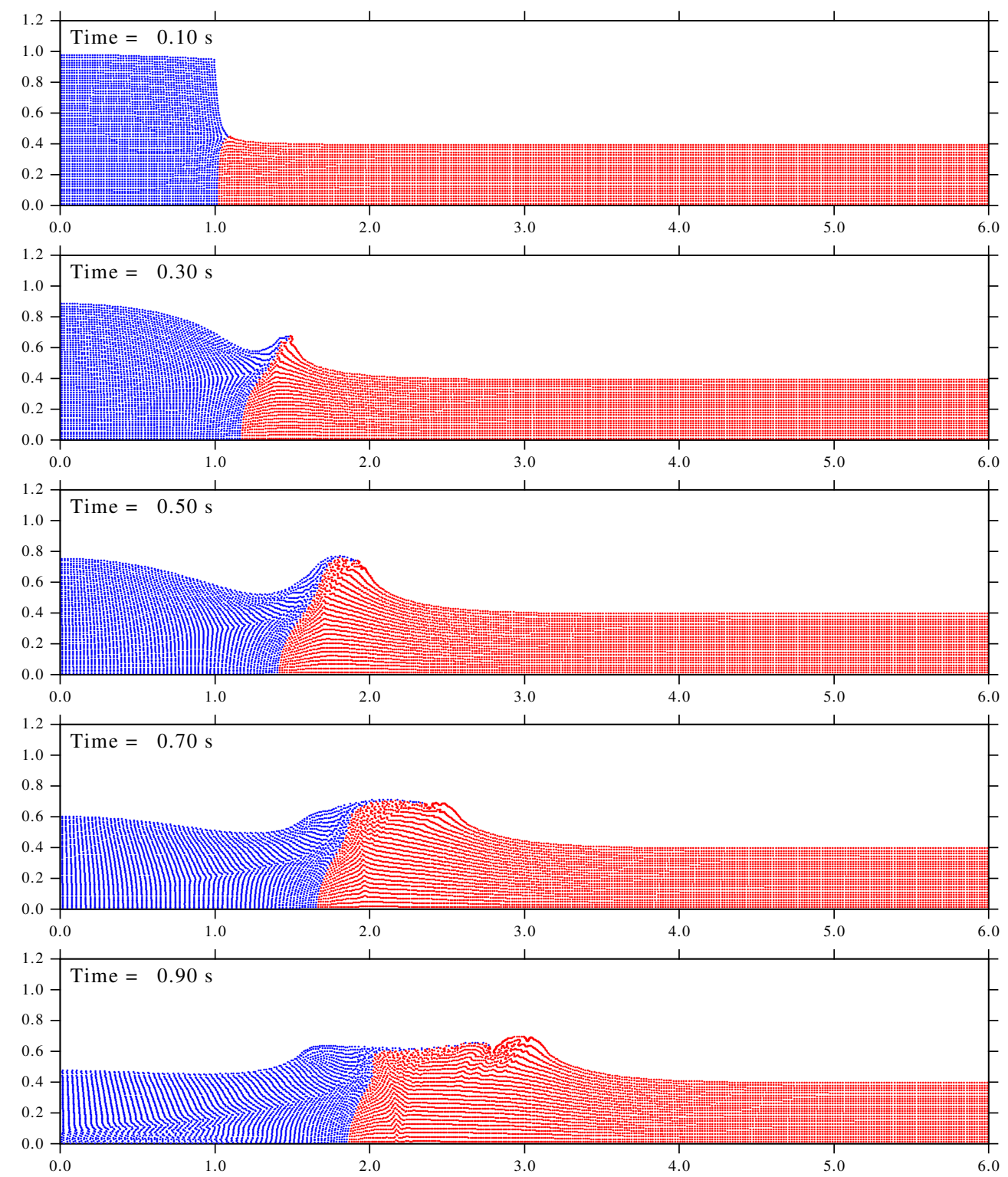

Fig. 6. Time evolution of the water domain during dam-break flow over a dry bed ( $L=H=1 \mathrm{~m}, H_{1}=0.4 \mathrm{~m}, L_{1}=5 \mathrm{~m}, 10800$ discrete particles used in the simulations)

see Figure 5. In the case of the deeper layer, illustrated in Figure 6, the progressive wave also develops, but its evolution is less violent and no wave breaking occurs.

Corresponding to the flow case shown in Figure 5 are the plots presented in Figures 7 and 8, which illustrate the evolution of water pressure and horizontal velocity fields during the wave-breaking process. The pressures and velocities in the plots are given in dimensionless forms, obtained by scaling the respective quantities by the 

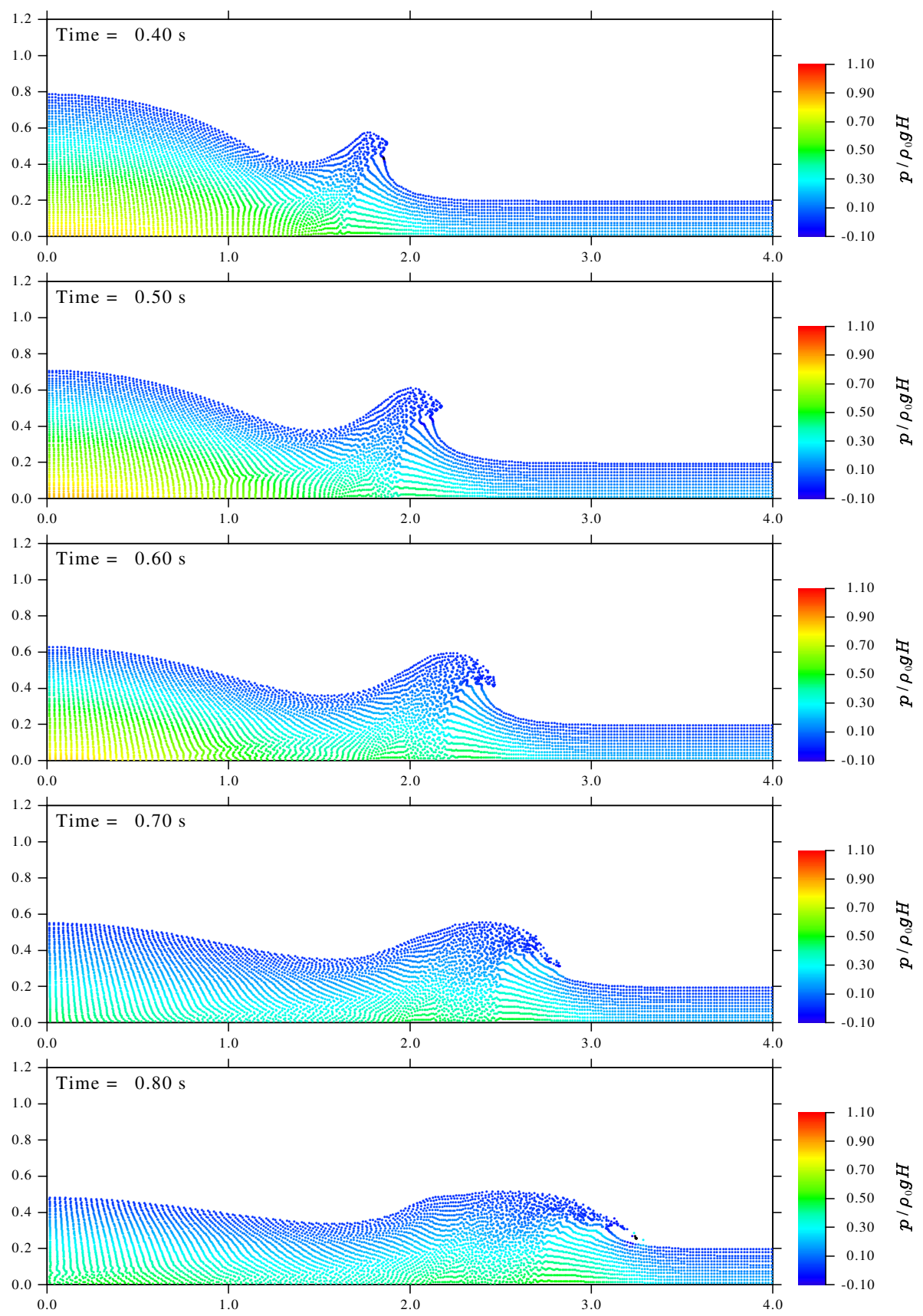

Fig. 7. Time evolution of dimensionless pressure field during dam-break flow over a water layer ( $L=H=1 \mathrm{~m}, H_{1}=0.2 \mathrm{~m}, L_{1}=3 \mathrm{~m}, 5760$ discrete particles used in the simulations) Particles are coloured by their velocities 


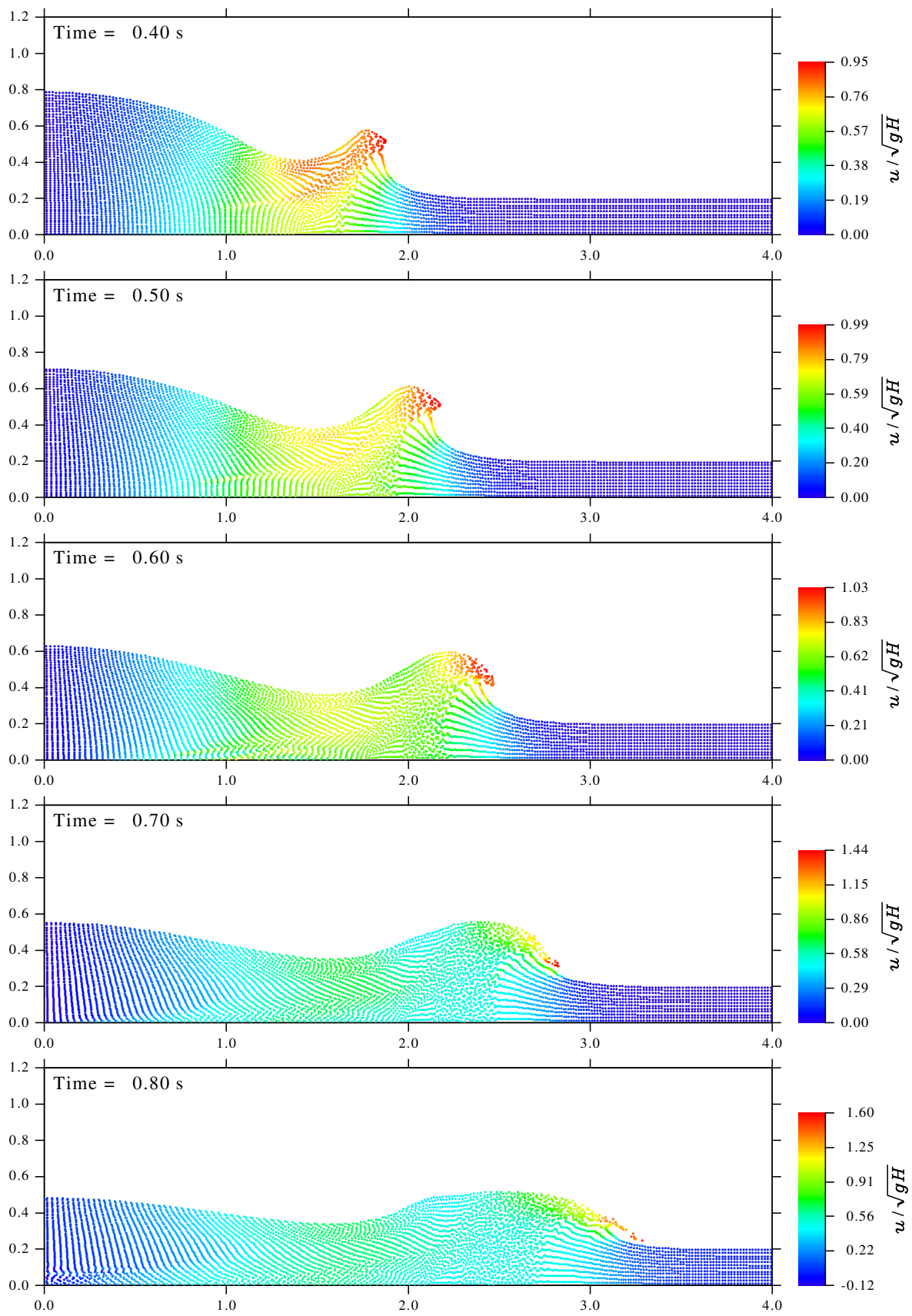

Fig. 8. Time evolution of dimensionless horizontal velocities during dam-break flow over a water layer $\left(L=H=1 \mathrm{~m}, H_{1}=0.2 \mathrm{~m}, L_{1}=3 \mathrm{~m}, 5760\right.$ discrete particles used in the simulations). Particles are coloured by their velocities 
units $\rho_{0} g H$ and $\sqrt{g H}$. It is seen that the discrete representations of the two fields are relatively smooth and regular in nearly the whole fluid domain. Some irregularities and numerical oscillations appear on the free surface directly ahead of the advancing water front only after a plunging jet has formed, followed by its disintegration due to the impact on the water surface below.
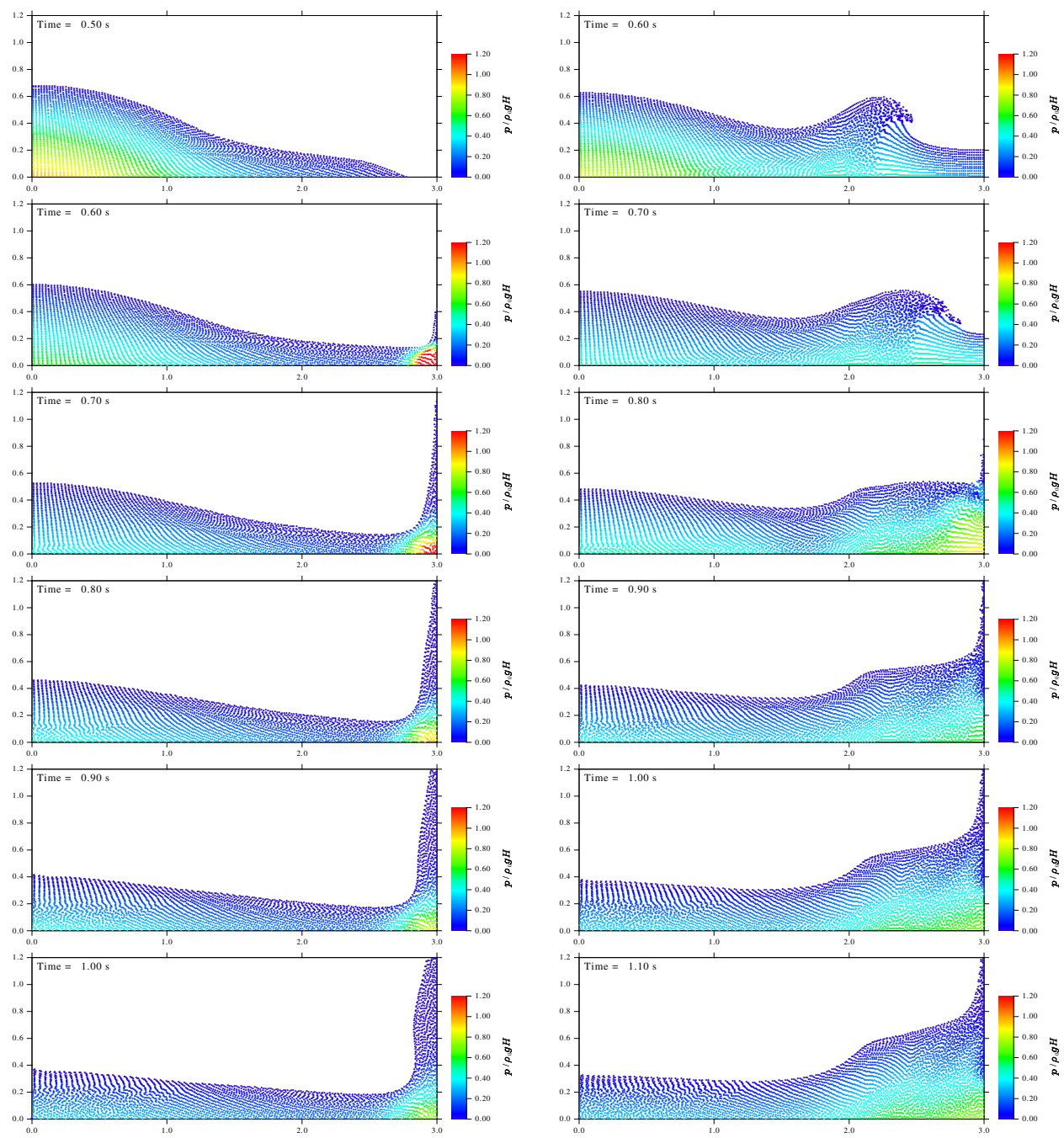

Fig. 9. Time evolution of dimensionless pressure field during dam-break flow over a dry bed (left column) and over a water layer (right column), for $L=H=1 \mathrm{~m}, L_{1}=2 \mathrm{~m}$ and $H_{1}=0.2 \mathrm{~m}$.

Particles are coloured by their pressures

Finally, a situation in which a surging water wave generated by the dam break hits a rigid vertical wall was simulated to determine the pressures exerted by water on the structure. The two cases sketched in Figure 2 were considered, with the initial fluid 
configurations defined by the parameters $L=H=1 \mathrm{~m}, L_{1}=2 \mathrm{~m}$ and $H_{1}=0.2 \mathrm{~m}$. The results obtained are presented in Figure 9. The consecutive plots in the left panel illustrate flow over a dry bed, whereas the plots on the right side show the pressure evolution in the case of flow in the presence of a water layer. One can see again that, even though water pressures vary rapidly, the ISPH model predicts reasonably smooth pressure fields. It follows from the plots that the maximum water impact pressures generated during flow over a dry bed significantly exceed those appearing in the presence of a water layer.
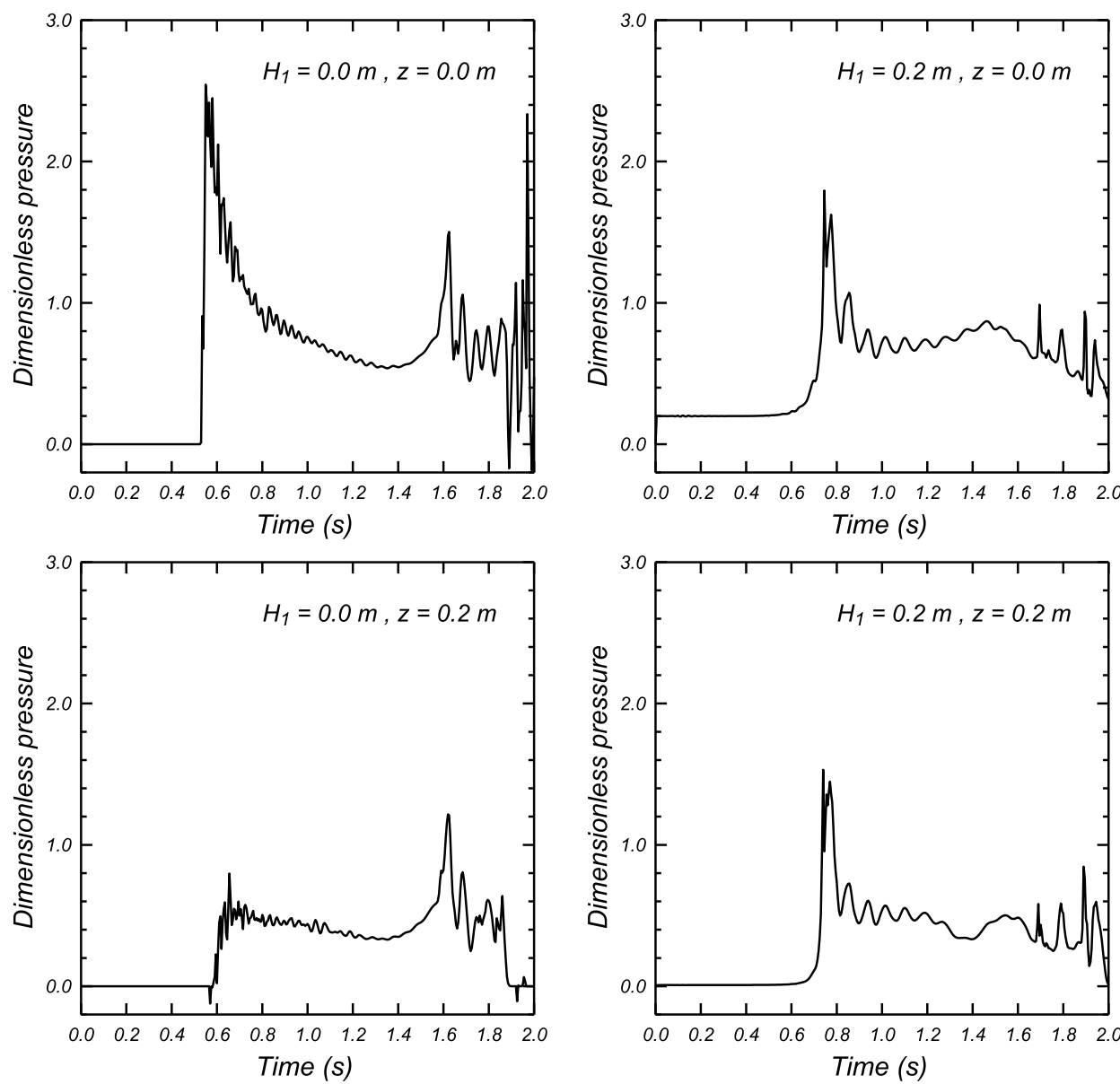

Fig. 10. Time history of dimensionless pressure $p / \rho_{0} g H$ exerted by a dam-break generated wave impacting on a rigid wall. Left column: flow over a dry bed $\left(H_{1}=0\right)$, right column: flow over a layer of water of depth $H_{1}=0.2 \mathrm{~m}$. The $z$-coordinates in the plots indicate the locations of the points on the wall for which the graphs are presented

The time variation of pressures exerted by a dam-break generated wave on a rigid wall is illustrated in Figure 10, showing pressure histories at two selected points: one 
at the bottom of the wall $(z=0)$ and the other at a height of $z=0.2 \mathrm{~m}$. Again, two flow cases are considered: flow over a dry bed, that is for $H_{1}=0$, and flow over a water layer of depth $H_{1}=0.2 \mathrm{~m}$. One can see that the pressures at the rigid wall change very rapidly and in an irregular manner from the instant of the first contact of the wave with the structure, reflecting the violent dynamics of the impact mechanism. Such behaviour has been observed in experiments, see, for instance, Colagrossi and Landrini (2003) and Rafiee et al (2012). Clearly, the largest pressures are exerted on the wall during water flow over a dry bed, with the peak values of about $2.5 \rho_{0} g H$ occurring at the bottom corner point $z=0$ (see the top left plot). The corresponding impact pressures at the point $z=0.2 \mathrm{~m}$ are much smaller, indicating that the main thrust of the surging wave is restricted to the near-bottom region. In contrast, in the case of a breaking wave impact illustrated in the two plots in the right column, the pressures at the points $z=0$ and $z=0.2 \mathrm{~m}$ (the initial free surface elevation of water in the channel) attain similar values (around $1.8 \rho_{0} g H$ at the bottom). Furthermore, the pressure variations in time at the two points are qualitatively similar.
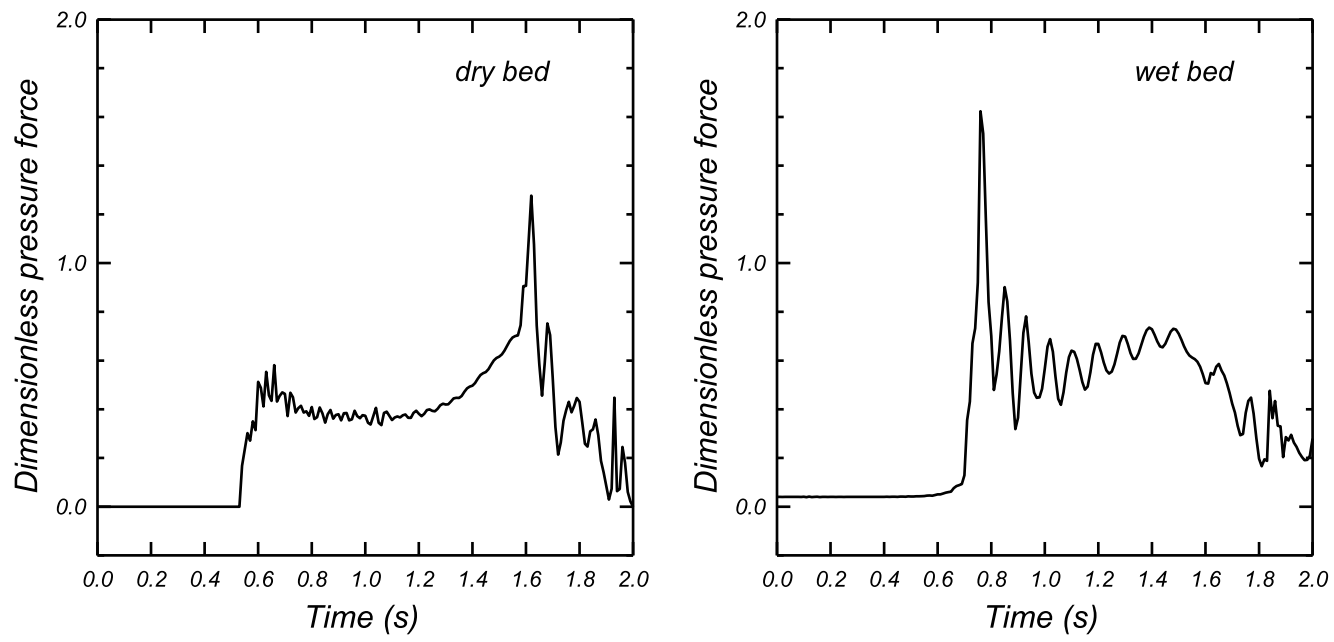

Fig. 11. Time history of dimensionless dynamic pressure force $P_{\text {dyn }} / P_{\text {stat }}\left(P_{\text {stat }}=1 / 2 \rho_{0} g H^{2}\right)$ exerted by a dam-break wave impacting on a rigid wall. Left plot: flow over a dry bed $\left(H_{1}=0\right)$, right plot: flow over a layer of water of depth $H_{1}=0.2 \mathrm{~m}$

Corresponding to the previous plots are those presented in Figure 11, illustrating the time variation of total dynamic pressure forces $P_{d y n}$ exerted by an impacting water wave on the vertical wall; these forces are calculated by integrating fluid pressures at all discrete particles being currently on the wall surface. The resulting dynamic forces are normalized by means of a static pressure force $P_{\text {stat }}=\frac{1}{2} \rho_{0} g H^{2}$, which is the total force exerted on the wall by still water of depth $H$. Again, two cases are considered, namely flow over a dry bed (the plot on the left), and flow over a wet bed, that is, over a layer of water of the initial depth $H_{1}=0.2 \mathrm{~m}$ (the plot on the right). It can be seen that 
in both cases the variations in time of the normalized total forces $P_{d y n} / P_{\text {stat }}$ resemble qualitatively the corresponding variations of the normalized dynamic pressures at the wall point $z=0.2 \mathrm{~m}$ (see the two plots in the lower row in Figure 10).

\section{Conclusions}

In this paper, the incompressible smoothed particle hydrodynamics (ISPH) method has been applied to solve numerically the equations describing violent free-surface water flow under gravity. The comparisons of the results of calculations with experimental data have shown that the ISPH method yields better quantitative predictions of the surging wave front position and of the water free-surface elevation than the standard, weakly compressible, SPH approach. However, the improvement has been achieved at a significantly greater computational cost, associated with the need to solve the Poisson equation for water pressures. In the simulations carried out here, this cost increased by a factor of about five. The plots illustrating the evolution of the water domain during a dam-break event prove that the numerical model is capable of realistically capturing physical phenomena taking place during violent transient water flows. The plots showing the variation of pressure and velocity fields remain smooth over long time scales, except those regions of water near its free surface in which wave breaking and jet formation occur. A more successful treatment of these phenomena may require, on one hand, consideration of additional physical mechanisms, such as surface-tension effects in the case of small geometrical scales, and, on the other hand, an increased resolution of the discrete model and the application of a more sophisticated numerical time-integration scheme.

\section{References}

Antoci C., Gallati M. and Sibilla S. (2007) Numerical simulation of fluid-structure interaction by SPH, Comput. Struct., 85 (11-14), 879-890, DOI: 10.1016/j.compstruc.2007.01.002.

Belytschko T., Krongauz Y., Dolbow J. and Gerlach C. (1998) On the completeness of meshfree particle methods, Inter. J. Numer. Meth. Eng., 43 (5), 785-819, DOI: 10.1002/(SICI)1097-0207(19981115) 43:5.

Belytschko T., Krongauz Y., Organ D., Fleming M. and Krysl P. (1996) Meshless methods: An overview and recent developments, Comput. Meth. Appl. Mech. Eng., 139 (1-4), 3-47.

Braess H. and Wriggers P. (2000), Arbitrary Lagrangian Eulerian finite element analysis of free surface flows, Comput. Meth. Appl. Mech. Eng., 190 (1-2), 95-109.

Chang T. J., Kao H. M., Chang K. H. and Hsu M. H. (2011) Numerical simulation of shallow-water dam break in open channels using smoothed particle hydrodynamics, J. Hydrol., 408 (1-2), 78-90, DOI: $10.1016 /$ j.hydrol.2011.07.023.

Chorin A. j. (1968) Numerical solution of the Navier-Stokes equations, Math. Comput., 22 (104), $745-762$.

Colagrossi A. and Landrini M. (2003) Numerical simulation of interfacial flows by smoothed particle hydrodynamics, J. Comput. Phys., 191 (2), 448-475, DOI: 10.1016/S0021-9991(03)00324-3.

Cummins S. J. and Rudman M. (1999) An SPH projection method, J. Comput. Phys., 152 (2), 584-607, DOI: $10.1016 / \mathrm{jcph} 1999.6246$. 
Cummins S. J., Silvester T. B. and Cleary P. W. (2012) Three-dimensional wave impact on a rigid structure using smoothed particle hydrodynamics, Int. J. Numer. Meth. Fluids, 68 (12), 1471-1496, DOI: $10.1016 /$ fld.2539.

Dalrymple R. A. and Rogers B. D. (2006) Numerical modeling of water waves with the SPH method, Coastal Eng., 53 (2-3), 141-147, DOI: 10.1016/j.coastaleng.2005.10.004.

Gingold R. A. and Monaghan J. J. (1977) Smoothed particle hydrodynamics: theory and application to non-spherical stars, Mon. Not. R. Astron. Soc., 181, 375-389.

Gómez-Gesteira M., Cerqueiro D., Crespo C. and Dalrymple R. A. (2005) Green water overtopping analyzed with a SPH method, Ocean Eng., 32 (2), 223-238, DOI: 10.1016/j.oceaneng.2004.08.003.

Harlow F. H. (2004) Fluid dynamics in Group T-3 Los Alamos National Laboratory (LA-UR-03-3852), J. Comput. Phys., 195 (2), 414-433, DOI: 10.1016/jcph2003.09.030.

Harlow F. H. and Welch J. E. (1965) Numericl calculation of time-dependent viscous incompressible flow of fluid with free surface, Phys. Fluids, 8 (12), 2182-2189.

Hu X. Y. and Adams N. A. (2007) An incompressible multi-phase SPH method, J. Comput. Phys., 227 (1), 264-278, DOI: 10.1016/j.jcp2007.07.013.

Johnson G. R., Stryk R. A. and Beissel S. R. (1996) SPH for high velocity impact computations, Comput. Appl. Mech. Eng., 139 (1-4), 347-373.

Li S. and Liu W. K. (2004) Meshfree Particle Methods, Springer, Berlin.

Lo E. Y. M. and Shao S. (2002) Simulation of near-shore solitary wave mechanics by an incompressible SPH method, Appl. Ocean Res., 24 (5), 275-286, DOI: 10.1016/S0141-1187(03)00002-6.

Lucy L. B. (1977) A numerical approach to the testing of the fission hypothesis, Astron. J., 82 (12), 1013-1024.

Martin J. C. and Moyce W. J. (1952) An experimental study of the collapse of liquid columns on a rigid horizontal plane, Phil. Trans. R. Soc. Lond. A 244 (882), 312-324.

Monaghan J. J. (1992) Smoothed particle hydrodynamics, Annu. Rev. Astron. Astrophys., 30, 543-574, DOI: 10.1146/annurev.aa.30.090192.002551.

Monaghan J. J. (1996) Gravity currents and solitary waves, Physica D, 98 (2-4), 523-533.

Morris J. P. (1996) Analysis of Smoothed Particle Hydrodynamics with Applications, Ph. D. thesis, Monash University, Melbourne, Australia.

Quecedo M., Pastor M., Herreros M. I., Fernández Merodo J. A. and Zhang Q. (2005) Comparison of two mathematical models for solving the dam break problem using the FEM method, Comput. Meth. Appl. Mech. Eng., 194 (36-38), 3984-4005, DOI: 10.1146/j.cma.2004.08.011.

Rabier S. and Medale M. (2003) Computation of free surface flows with a projection FEM in a moving mesh framework, Comput. Meth. Appl. Mech. Eng., 192 (41-42), 4703-4721.

Radovitzky R. and Ortiz M. (1998) Lagrangian finite element analysis of Newtonian viscous flow, Int. J. Numer. Meth. Eng., 43 (4), 608-619.

Rafiee A., Cummins S., Rudman M. and Thiagarajan K. (2012) Comparative study on the accuracy and stability of SPH schemes in simulating energetic free-surface flows, Eur. J. Mech. B/Fluids, 36 (1-16), DOI: 10.1146/j.euromechflu.2012.05.001.

Ramaswamy B. and Kawahara M. (1987) Lagrangian finite element analysis applied to viscous free surface flow, Int. J. Numer. Meth. Fluids, 7 (9), 953-984.

Randles P. W. and Libersky L. D. (1996) Smoothed Particle Hydrodynamics. Some recent improvements and applications, Comput. Meth. Appl. Mech. Eng., 139 (1-4), 375-408.

Shao S. (2006) Incompressible SPH simulation of wave breaking and overtopping with turbulence modelling, Int. J. Numer. Meth. Fluids, 50 (5), 597-621, DOI: 10.1002/fld.1068.

Shao S. (2010) Incompressible SPH flow model for wave interactions with porous media, Coastal Eng., 57 (3), 304-316, DOI: 10.1016/j.coastaleng.2009.10.012. 
Shao S. and Lo E. Y. M. (2003) Incompressible SPH for simulating Newtonian and non-Newtonian flows with a free surface, Adv. Water Resour., 26 (7), 787-800, DOI: 10.1016/S0309-1708(03)0030-7.

Souli M. and Zolesio J. P. (2001) Arbitrary Lagrangian-Eulerian and free surface methods in fluid mechanics, Comput. Meth. Appl. Mech. Eng., 191 (3-5), 451-466.

Staroszczyk R. (2007) A Lagrangian finite element treatment of transient gravitational waves in compressible viscous fluids, Arch. Hydro-Eng. Environ. Mech., 54 (4), 261-284.

Staroszczyk R. (2009) A Lagrangian finite element analysis of gravity waves in water of variable depth, Arch. Hydro-Eng. Environ. Mech., 56 (1-2), 43-61.

Staroszczyk R. (2010) Simulation of dam-break flow by a corrected smoothed particle hydrodynamics method, Arch. Hydro-Eng. Environ. Mech., 57 (1), 61-79.

Staroszczyk R. (2011) Simulation of solitary waves mechanics by a corrected smoothed particle hydrodynamics method, Arch. Hydro-Eng. Environ. Mech., 58 (1-4), 23-45, DOI: 10.2478/ v10203-011-0002-9.

Szydłowski M. and Zima P. (2006) Two-dimensional vertical Reynolds-averaged Navier-Stokes equations versus one-dimensional Saint-Venant model for rapidly varied open channel water flow modelling, Arch. Hydro-Eng. Environ. Mech., 53 (4), 295-309. 